\title{
Abnormal Myocardial Capillary Density in Apical Hypertrophic Cardiomyopathy Can Be Assessed by Myocardial Contrast Echocardiography
}

\author{
Jeonggeun Moon, MD; In Jeong Cho, MD; Chi Young Shim, MD, PhD; \\ Jong-Won Ha, MD, PhD; Yangsoo Jang, MD, PhD; \\ Namsik Chung, MD, PhD; Se-Joong Rim, MD, PhD
}

\begin{abstract}
Background: Myocardial ischemia and dysfunction can occur in hypertrophic cardiomyopathy (HCM) because of the high muscle-to-blood ratio, even without significant coronary artery disease. Microbubbles reside only in the intravascular space and myocardial video-intensity during systole results mostly from microbubbles within capillaries. The hypothesis explored in the present study was that an abnormal capillary density in apical HCM $(\mathrm{ApHCM})$ can be demonstrated using myocardial contrast echocardiography (MCE).
\end{abstract}

Methods and Results: The 56 patients were investigated (31 males, age 58 \pm 9 years; 33 ApHCM, 9 hypertensive left ventricular hypertrophy [LVH], 14 controls). MCE was performed with low-mechanical-index power modulation imaging. Tissue Doppler imaging to assess myocardial contractile function was obtained at the mitral

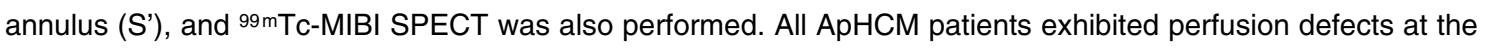
hypertrophied segments in the systolic phase during MCE, whereas SPECT showed normal or rather increased perfusion at those sites. The cyclic variation of video-intensity was exaggerated in ApHCM when compared with the LVH or control group (\% of [systolic video-intensity]/[diastolic video-intensity]: $33.0 \pm 12.3 \%, 88.3 \pm 19.2 \%$ and $79.4 \pm 13.9 \%$, respectively $[P<0.05])$. Concurrently, MCE cyclic variation and perfusion defect size were related to decreased $S^{\prime}(P<0.05$ for all).

Conclusions: A perfusion defect at the hypertrophied segment, representing abnormal myocardial capillary density, was observed in ApHCM patients during MCE. The extent of MCE cyclic variation and the perfusion defect size both correlate with decreased myocardial contractile property in ApHCM. (Circ J 2010; 74: 21662172)

Key Words: Capillary density; Contrast echocardiography; Hypertrophic cardiomyopathy

$\mathbf{H}$ ypertrophic cardiomyopathy (HCM) is clinically diagnosed by the presence of unexplained left ventricular hypertrophy (LVH), and pathologically diagnosed by myocyte hypertrophy, disarray, and interstitial fibrosis. ${ }^{1,2}$ Apical HCM (ApHCM) is a variant of HCM, characterized by myocardial hypertrophy occurring predominantly in the left ventricular (LV) apex, with an "ace of spade" configuration of the LV and giant negative T-wave on ECG. ${ }^{3}$ A decrease in coronary flow reserve associated with narrowing of the small intramyocardial arteries, despite usually normal epicardial coronary arteries, has been known to occur in patients with ApHCM. Although the exact cause of the decreased blood flow is still in some doubt, it may be related to small-vessel disease with decreased vasodilator capacity. ${ }^{4-6}$ At the same time, it has been reported that capillary density is reduced in HCM. ${ }^{7}$ Such histologic changes and resultant microvascular ischemia are thought to be factors related to the impaired myocardial contraction and relaxation observed in HCM. However, there is no clinically applicable non-invasive diagnostic modality for assessing reduced capillary density in the hypertrophied segment and its degree in these patients.

Myocardial contrast echocardiography (MCE) is a novel technique that uses microbubbles to produce myocardial opacification. Microbubbles have intravascular rheologic characteristics similar to those of erythrocytes and reside

Received March 15, 2010; revised manuscript received June 13, 2010; accepted June 14, 2010; released online August 31, 2010 Time for primary review: 36 days

Cardiology Division, Yonsei Cardiovascular Center, Yonsei University College of Medicine, Seoul (J.M., I.J.C., C.Y.S., J.-W.H., Y.J., N.C.); Cardiology Division, Department of Internal Medicine, Gangnam Severance Hospital, Yonsei University College of Medicine, Seoul (S.-J.R.), Republic of Korea

Mailing address: Se-Joong Rim, MD, PhD, Cardiology Division, Department of Internal Medicine, Gangnam Severance Hospital, Yonsei University College of Medicine, 712 Eonjuro, Gangnam-gu, Seoul 135-270, Republic of Korea. E-mail: sejoong@yuhs.ac

ISSN-1346-9843 doi:10.1253/circj.CJ-10-0241

All rights are reserved to the Japanese Circulation Society. For permissions, please e-mail: cj@j-circ.or.jp 
only in the intravascular space. ${ }^{8-10}$ Therefore, the microbubble concentration (video-intensity in MCE) represents the relative blood volume of the myocardium. Furthermore, myocardial video-intensity during systole results mostly from microbubbles within myocardial capillaries, because blood in the small- to medium-sized arterioles and venules in the myocardium is squeezed out in the systolic phase. ${ }^{11,12}$ Our hypothesis in the present study was that an abnormally decreased capillary density and reduced blood volume of the hypertrophied myocardium associated with a high muscleto-blood ratio in ApHCM can be demonstrated using MCE. Simultaneously, we investigated the correlation of MCE cyclic variation and perfusion defect size with myocardial contractile properties assessed by tissue Doppler imaging (TDI).

\section{Methods}

\section{Study Population}

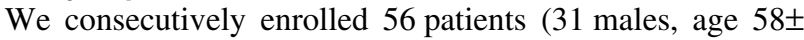
9 years; 33 ApHCM, 9 hypertensive LVH, 14 normal LV) with no clinical evidence of coronary artery disease (CAD) (Severance Hospital, Yonsei University Medical Center, Seoul, Republic of Korea). Patients with ischemic heart disease, atrial fibrillation or left bundle-branch block on ECG, primary valvular heart disease, regional wall-motion abnormalities, or an LV ejection fraction (EF) less than $50 \%$ on echocardiography were excluded. The diagnosis of HCM was made according to the World Health Organization/International Society and Federation of Cardiology criteria. ${ }^{13}$ All ApHCM patients had giant negative T-waves in the precordial ECG leads, a characteristic 'ace of spades' configuration of the $\mathrm{LV}$, and no intraventricular pressure gradient on echocardiographic evaluation. History of hypertension (HTN) was defined as: (1) patient previously diagnosed as having HTN and prescribed antihypertensive medication, and (2) patient demonstrates resting systolic blood pressure $\geq 140 \mathrm{mmHg}$ or resting diastolic blood pressure $\geq 90 \mathrm{mmHg}$ on 2 measurements performed at 5-min intervals in a quiet room. Nine patients with hypertensive LVH had both HTN and LVH determined by echocardiography. ${ }^{14}$ This study was approved by the Institutional Review Board and complied with the Declaration of Helsinki. Informed consent was given by all study subjects.

\section{Echocardiographic Evaluation}

A comprehensive transthoracic echocardiographic evaluation was performed in all patients at the time of initial diagnosis before MCE was performed. Standard M-mode, 2-dimensional, and color Doppler images were obtained in the parasternal and apical views. End-diastole was defined as the frame with the largest cavity area immediately before the onset of the QRS, and end-systole as the frame with the smallest cavity area. LV end-diastolic volume, LV end-systolic volume, and LVEF were calculated from 2-D recordings using the modified biplane Simpson's method as described previously..$^{15} \mathrm{LV}$ mass was calculated as:

\section{$0.8\left[1.04(\text { SWTd }+ \text { LVIDd + PWTd })^{3}-\right.$ LVIDd $\left.^{3}\right]+0.6(\mathrm{~g})$}

where LVIDd is the internal diameter of LV at end-diastole and PWTd and SWTd are respectively the posterior wall thickness and septal wall thickness at end-diastole. ${ }^{14}$ The LV mass was indexed to body surface area (LV mass index). Left atrial (LA) volume was assessed by the biplane arealength method and was indexed to body surface area (LA volume index [LAVI]). ${ }^{14}$ All patients underwent an evaluation with pulsed-wave Doppler echocardiography of mitral inflow and TDI. From the mitral inflow velocities, the following variables were measured: peak velocity of early diastolic filling (E), late filling (A), and deceleration time of the E-wave velocity. On TDI, peak systolic mitral annular velocity (S') and early diastolic mitral annular velocity (E') were measured from the apical 4-chamber view with a 2- to 5-mm sample volume placed at the septal and lateral corner of the mitral annulus. The mean of 3-6 measurements was used for analysis. E/E' was calculated to estimate LV filling pressure. Right ventricular systolic pressure was calculated from tricuspid regurgitant flow velocity as determined in continuous wave Doppler mode.

\section{MCE Using Perfluorocarbon-Exposed Sonicated Dextrose Albumin (PESDA)}

MCE was performed at rest, with low-mechanical-index $(<0.1)$ power modulation imaging (Sonos 5500, Agilent, USA) during continuous infusion of microbubbles. PESDA was prepared in a manner similar to previously described methods: ${ }^{16}$ $5 \%$ percent human serum albumin and 5\% dextrose were used to prepare the microbubbles. Next, $12 \mathrm{ml}$ of $5 \%$ dextrose and $4 \mathrm{ml}$ of $5 \%$ human serum albumin (total $16 \mathrm{ml}$ ) were drawn into a 35-ml monojet syringe. Each dextrose albumin sample was hand-agitated with $8 \mathrm{ml}$ of perfluorocarbon (molecular weight: $188 \mathrm{~g} / \mathrm{mol}$ ), and the sample was then exposed to eletromechanical sonification for 80 s using commercially available equipment (Heat System Inc, USA). According to the manufacturer, the sonification process involves the transference of electrical energy to mechanical energy (495 W). Energy output was adjusted by means of a digital scale during the sonification process to achieve $25 \pm 3 \%$ of this maximal output during the entire sonification time $(124 \pm 15 \mathrm{~W})$. Because the energy is emitted vertically from the circular 0.5-inch horn tip, the output from the horn tip was estimated to be $98 \pm 11 \mathrm{~W} / \mathrm{cm}^{2}$. The mean microbubble size of PESDA made by this method is known to be $4.7 \pm 0.2 \mu \mathrm{m}$, with a mean concentration of $1.3 \pm 0.1 \times 10^{9}$ microbubbles $/ \mathrm{ml}^{16}$ PESDA $(0.00125-0.0025 \mathrm{ml} / \mathrm{kg})$ was injected into the antecubital vein through a 19-gauge needle. MCE images were then obtained under resting conditions with the constant gain setting determined most appropriately before starting imaging. Images were acquired from the apical 4-, 3- and 2-chamber views. The region of interest was placed at the LV apex. Systolic video-intensity was determined at end-systole (the end of the T wave on ECG) and diastolic video-intensity was determined at end-diastole (appearance of the QRS complex on ECG). Cyclic variation of video-intensity (\% of [systolic video-intensity]/[diastolic video-intensity]) at the apex was measured using Yabco MCE software (Yabco, USA). Perfusion defect size (\% of [perfusion defect area]/[LV myocardial area] in apical view) was determined using Image J (NIH, USA). The echocardiographic data were analyzed by 2 experienced echocardiographers who were unaware of patients' clinical data.

\section{Sestamibi Single-Photon Emission Computed Tomography (99mTc-MIBI SPECT)}

99mTc-MIBI SPECT using a double headed camera was additionally performed for every enrolled patient to exclude significant $\mathrm{CAD}$ and to assess myocardial blood flow. Adenosine stress SPECT imaging was performed 45-60 min after the injection of 8-15 millicuries of MIBI through a dedicated intravenous line. Rest MIBI SPECT images were acquired 4-6h later using 25-35 millicuries MIBI. The images were 


\begin{tabular}{|c|c|c|c|c|}
\hline & $\begin{array}{c}\text { ApHCM } \\
(n=33)\end{array}$ & $\begin{array}{l}\text { Hypertensive LVH } \\
\qquad(n=9)\end{array}$ & $\begin{array}{c}\text { Normal subjects } \\
(n=14)\end{array}$ & P value * \\
\hline Age (years) & $57 \pm 10$ & $55 \pm 8$ & $61 \pm 9$ & 0.364 \\
\hline Male (n, \%) & $21(64)$ & $7(8)$ & $3(21)$ & 0.027 \\
\hline LVEF (\%) & $71.5 \pm 6.2$ & $71.5 \pm 7.2$ & $68.0 \pm 7.7$ & 0.357 \\
\hline LAVI (ml/m²) & $33.1 \pm 9.2$ & $20.0 \pm 5.8$ & $22.8 \pm 5.5$ & 0.004 \\
\hline LVEDD (mm) & $50.3 \pm 3.1$ & $46.1 \pm 5.8$ & $45.5 \pm 4.1$ & 0.002 \\
\hline LVESD (mm) & $31.0 \pm 3.3$ & $28.4 \pm 3.3$ & $29.1 \pm 3.7$ & 0.093 \\
\hline SWTd (mm) & $11.1 \pm 1.7$ & $12.6 \pm 2.9$ & $9.4 \pm 2.0$ & 0.005 \\
\hline SWTs (mm) & $15.1 \pm 2.0$ & $15.8 \pm 3.8$ & $12.9 \pm 3.0$ & 0.048 \\
\hline PWTd (mm) & $10.5 \pm 1.3$ & $12.1 \pm 2.7$ & $9.0 \pm 1.5$ & 0.001 \\
\hline PWTs (mm) & $14.8 \pm 1.6$ & $16.3 \pm 2.8$ & $12.6 \pm 2.3$ & 0.002 \\
\hline$E(\mathrm{~m} / \mathrm{s})$ & $0.60 \pm 0.16$ & $0.60 \pm 0.05$ & $0.60 \pm 0.13$ & 0.993 \\
\hline$A(m / s)$ & $0.63 \pm 0.15$ & $0.75 \pm 0.07$ & $0.68 \pm 0.16$ & 0.301 \\
\hline $\mathrm{DT}(\mathrm{ms})$ & $193.1 \pm 31.4$ & $235.3 \pm 37.7$ & $196.0 \pm 27.8$ & 0.057 \\
\hline $\mathrm{E}^{\prime}$, septal $(\mathrm{cm} / \mathrm{s})$ & $4.4 \pm 1.4$ & $5.2 \pm 1.5$ & $6.3 \pm 2.3$ & 0.053 \\
\hline $\mathrm{S}^{\prime}$, septal $(\mathrm{cm} / \mathrm{s})$ & $6.2 \pm 1.2$ & $7.5 \pm 1.5$ & $7.3 \pm 1.0$ & 0.008 \\
\hline $\mathrm{S}^{\prime}$, lateral $(\mathrm{cm} / \mathrm{s})$ & $6.6 \pm 1.8$ & $9.0 \pm 2.8$ & $9.1 \pm 1.0$ & $<0.0001$ \\
\hline$A^{\prime}$, septal $(\mathrm{cm} / \mathrm{s})$ & $7.6 \pm 2.0$ & $8.1 \pm 0.8$ & $8.9 \pm 1.5$ & 0.303 \\
\hline $\mathrm{E} / \mathrm{E}^{\prime}$ & $14.4 \pm 4.4$ & $12.4 \pm 3.8$ & $10.3 \pm 3.0$ & 0.096 \\
\hline RVSP (mmHg; measured by TR velocity) & $28.8 \pm 4.7$ & $26.8 \pm 8.1$ & $23.5 \pm 3.3$ & 0.313 \\
\hline
\end{tabular}

${ }^{*} \mathrm{P}<0.05$ considered significant.

ApHCM, apical hypertrophic cardiomyopathy; LVH, left ventricular hypertrophy; LVEF, left ventricular ejection fraction; LAVI, left atrial volume index; LVEDD, left ventricular end-diastolic dimension; LVESD, left ventricular end-systolic dimension; SWTd, end-diastolic septal wall thickness; SWTs, end-systolic septal wall thickness; PWTd, end-diastolic posterior wall thickness; PWTs, end-systolic posterior wall thickness; E, peak velocity of early diastolic filling; A, peak velocity of late diastolic filling; DT, deceleration time of E; E', early diastolic mitral annular velocity; S', peak systolic mitral annular velocity; A', late diastolic mitral annular velocity; E/E', early mitral inflow velocity to early diastolic mitral annular velocity ratio; RVSP, right ventricular systolic pressure; TR, tricuspid regurgitation.

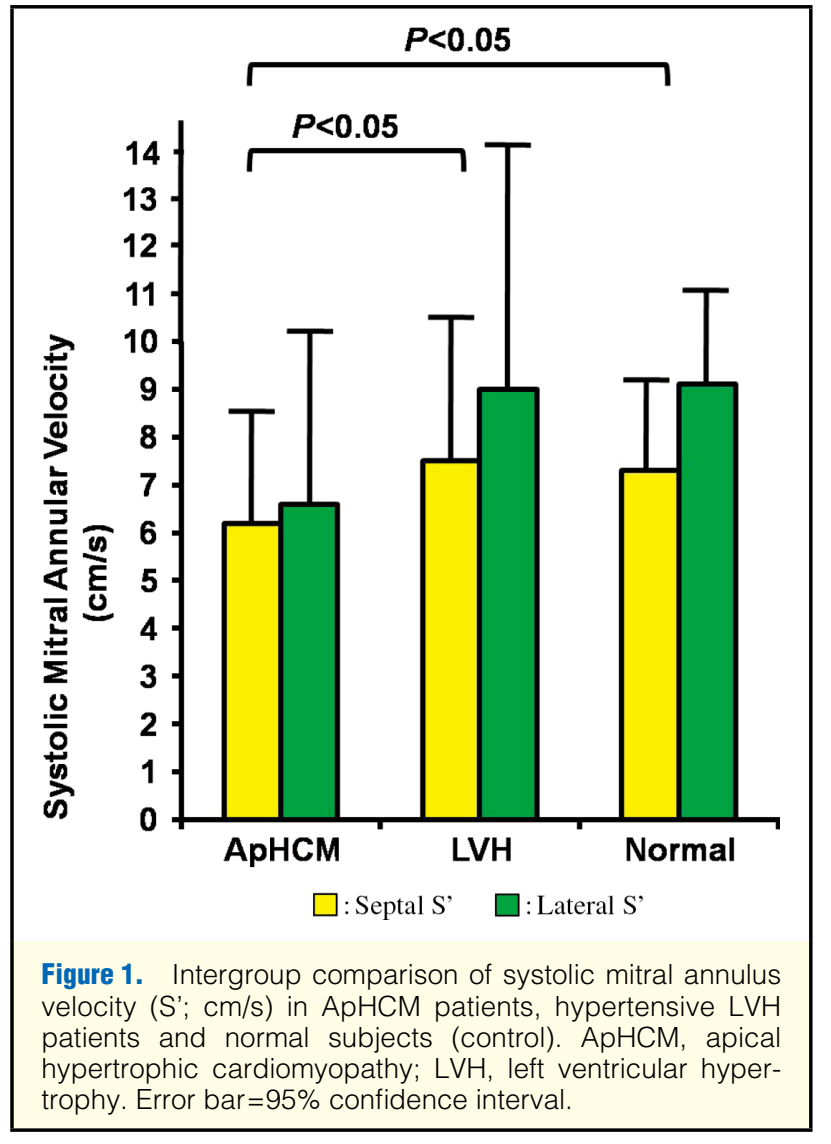

processed at the institution's nuclear laboratory and analyzed by 2 experienced observers who were unaware of the clinical and echocardiographic information of the patients.

\section{Statistical Analysis}

Continuous variables are listed as mean \pm SD when appropriate. Categorical variables are presented as frequencies and group percentages. Clinical and echocardiographic differences between the study groups were assessed using $\chi^{2}$ statistics and Fisher's exact test for categorical variables, and 1-way ANOVA test for continuous variables. Post hoc analyses were performed using the Bonferroni procedure. Pearson's bivariate correlation analysis was used to determine the correlation between variables. SPSS 12.0 (SPSS Inc, Chicago, IL, USA) was used for all statistical analyses. $\mathrm{P}<0.05$ was considered to be statistically significant.

\section{Results}

Clinical characteristics and echocardiographic parameters of the enrolled patients are shown in Table. The proportion of males was higher in the ApHCM group. LVEFs were grossly comparable among each group of subjects. However, $S$ ' values determined at the septal and lateral corner in patients with ApHCM were significantly lower than those of the hypertensive LVH group and the normal LV group $(\mathrm{P}<0.05$ for all). Post hoc analysis also showed that septal and lateral $S$ ' were significantly decreased in patients with ApHCM, when compared separately with those of the hypertensive LVH group or the normal LV group (Figure 1, $\mathrm{P}<0.05$ for all). At the same time, LAVI was significantly higher in ApHCM patients than in the hypertensive LVH and normal 
A
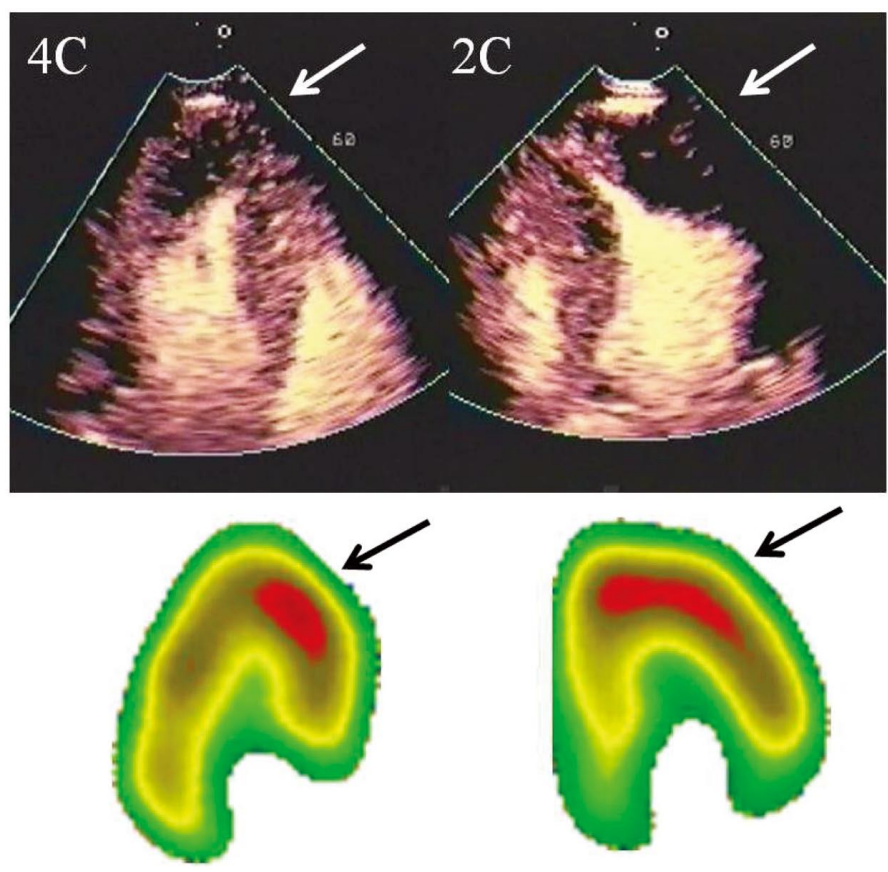

B
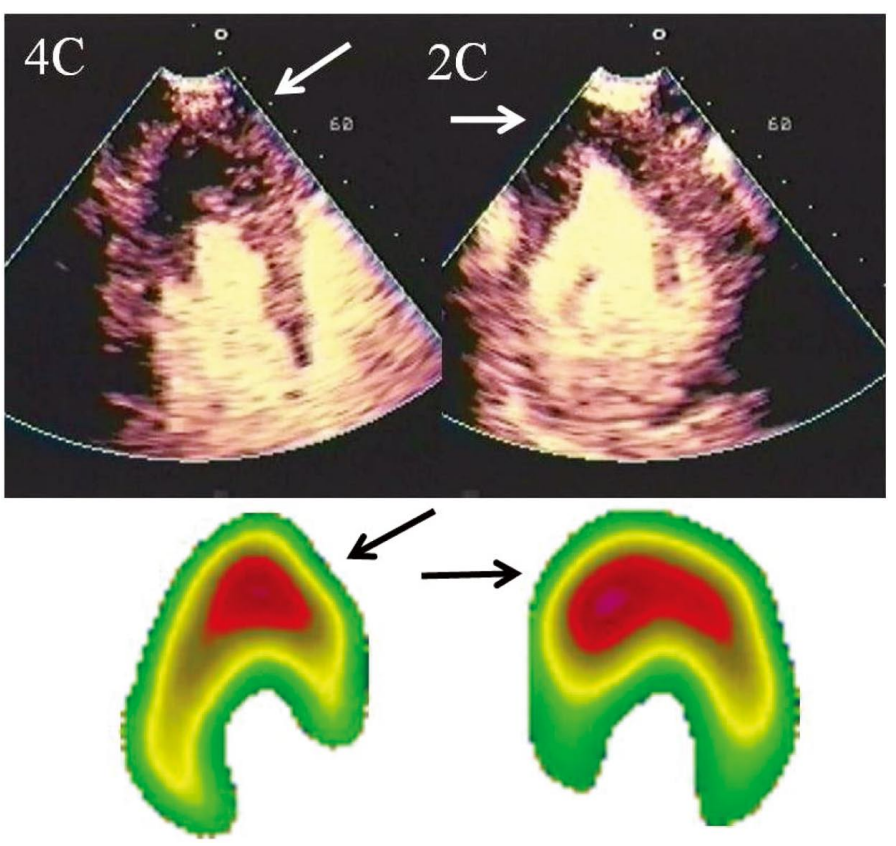

C

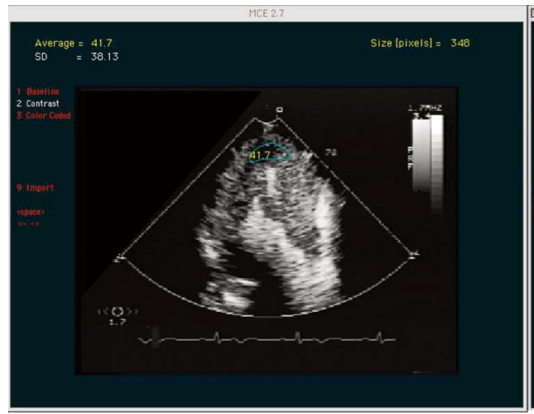

Systole

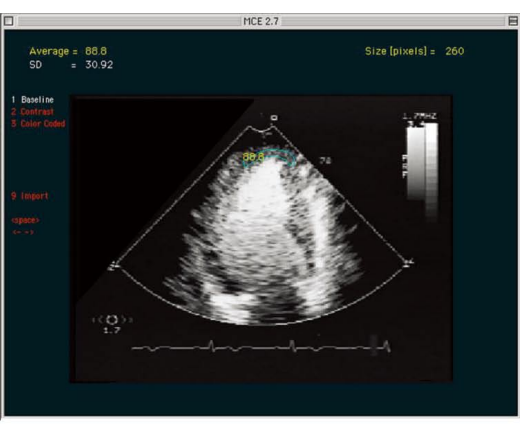

Diastole
Figure 2. Typical myocardial contrast echocardiography (MCE) and single-photon emission computed tomography (SPECT) images of apical hypertrophic cardiomyopathy in a 61-year-old male (A: cyclic variation of $37.6 \%$ ) and 62 year old male (B: cyclic variation of $21.6 \%$ ). 4C, 4-chamber view at end-systole; 2C, 2-chamber view at end-systole. Note the abnormal perfusion defect in apical segments during MCE (white arrows), whereas SPECT showed normal or rather increased perfusion of apical segments (black arrows). (C) Measuring cyclic variation at the apical lesion of left ventricle (\% of systolic video-intensity [41.7]/ diastolic video-intensity [88.8]= $47.0 \%)$. 


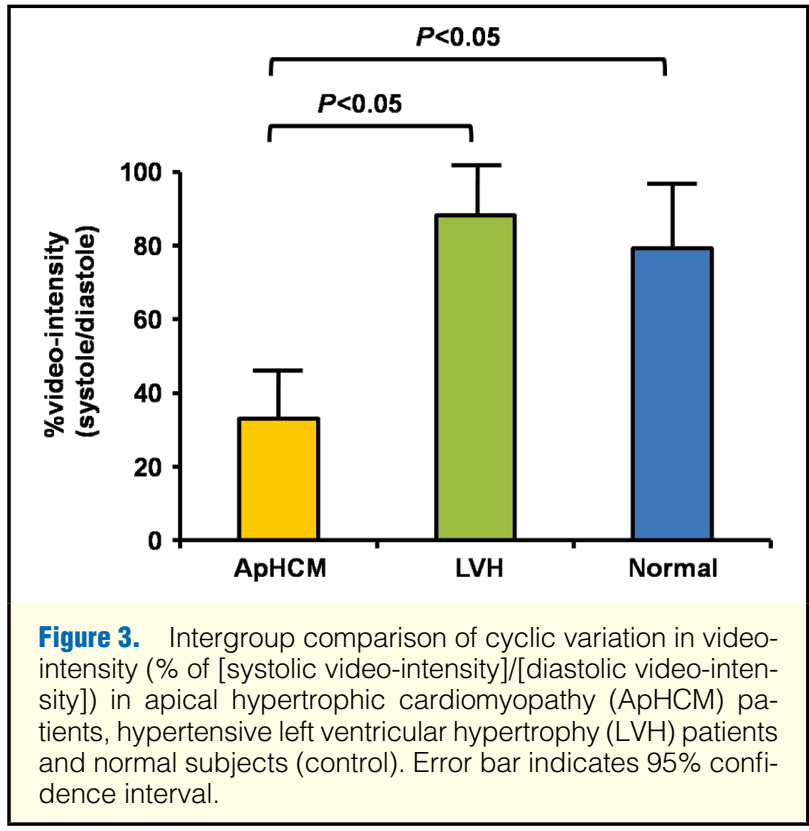

LV groups. The LV end-diastolic dimension was also significantly higher. SWTd, end-systolic septal wall thickness, PWTd, and end-systolic posterior wall thickness were significantly different among each group $(\mathrm{P}<0.05$ for all). In all patients with ApHCM, MCE showed an abnormal perfusion defect in the apical segments, whereas SPECT showed normal or rather increased perfusion of those portions. Figure 2 shows typical MCE findings in patients with ApHCM (Figures 2A, B) and measurements of the cyclic variation of video-intensity (Figure 2C). A decrease in the video-intensity during systole was observed at the apex in patients with ApHCM (Figure 3). The cyclic variation of video-intensity was significantly exaggerated in ApHCM when compared with LVH and normal LV $(33.0 \pm 12.3 \%, 88.3 \pm 19.2 \%$ and $79.4 \pm 13.9 \%$, respectively $[\mathrm{P}<0.05])$. MCE cyclic variation showed a significant positive correlation with S' (Figure 4 $[\mathrm{P}<0.05, \mathrm{r}=0.51])$. In patients with ApHCM, MCE defect size showed a significant negative correlation with S' (Figure 5 $[\mathrm{P}<0.05, \mathrm{r}=-0.47])$.
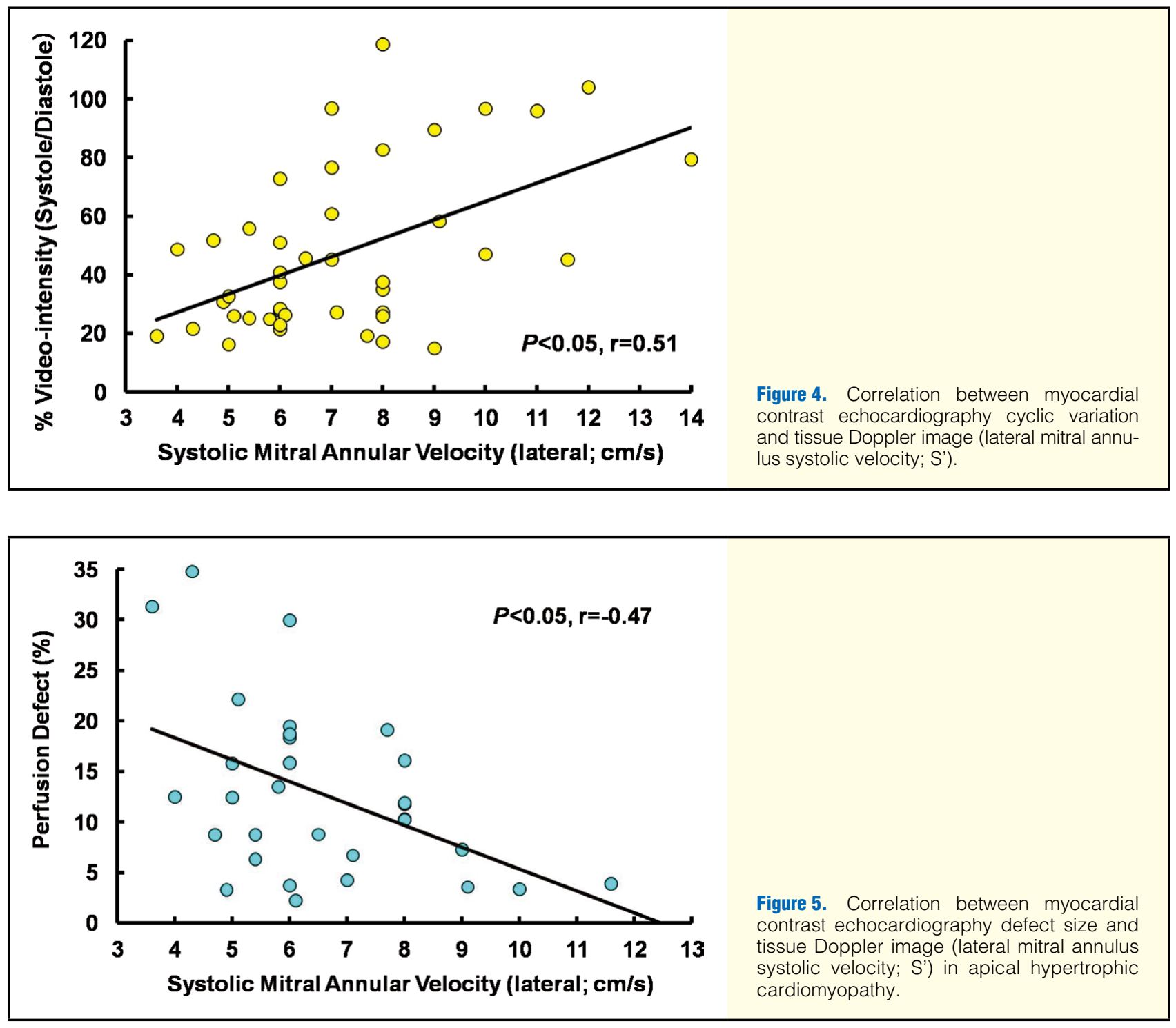


\section{Discussion}

The principal findings of the present study are that (1) a perfusion defect in the hypertrophied apical segment was observed using MCE in patients with ApHCM, and (2) the degree of MCE cyclic variation and the perfusion defect size were significantly correlated with decreased systolic mitral annular velocity.

\section{ApHCM and Microvascular Dysfunction}

Apical hypertrophy is a common type of HCM in East Asia and has been thought to have a relatively favorable prognosis. In recent years, however, the condition has been reported as less benign than previously considered. Severe apical hypertrophy could impose pressure overload and impair coronary flow in the LV apex, thereby inducing myocardial ischemia. ${ }^{17}$ Myocardial infarction appears to be a common complication of ApHCM during long-term follow-up, even without significant CAD. ${ }^{18}$ Microvascular dysfunction and structural changes, such as collagen deposition around penetrating intramyocardial coronary arteries in HCM, are thought to decrease coronary flow reserve in these patients. ${ }^{19}$ It is also well known that the volume of the interstitial collagen compartment is greatly increased in HCM. Furthermore, a decreased capillary density has been measured in several animal studies with experimentally induced secondary hypertrophy and it has also been reported that decreased coronary flow reserve in HCM is related to remodeling of the coronary microcirculation. ${ }^{7}$ This microvascular small-vessel disease is responsible for clinically silent myocardial ischemia, myocyte death, and repair in the form of replacement scars, which could serve as an electrically unstable substrate and nidus for reentry ventricular tachyarrhythmias. Recently, microvascular dysfunction in ApHCM was regarded as having a pivotal role in the clinical manifestations and prognosis of patients. Therefore, evaluation of reduced capillary density and microvascular dysfunction in ApHCM may be of clinical importance.

\section{MCE in ApHCM}

Clinically, it can be occasionally challenging to differentiate HCM from hypertensive heart disease and athletes hearts on the basis of morphologic or functional abnormalities alone. ${ }^{20}$ The results of the present study illustrate that reduced capillary density and decreased blood volume of the hypertrophied myocardial segment in ApHCM can be assessed non-invasively by MCE. The microbubble is a unique medium that resides only in the intravascular space, whereas the nuclear tracer used in ${ }^{99 \mathrm{~m} T c-M I B I}$ SPECT to evaluate CAD migrates to the interstitial or intracellular space of the myocardium. At the same time, small- to medium-sized arterioles and venules in the myocardium collapse and blood in those vessels is squeezed out during systole, ${ }^{11,12}$ while blood remains in myocardial capillaries. ${ }^{21}$ Thus, it can be said that myocardial video-intensity during systole results mostly from microbubbles within capillaries. On the other hand, myocardial video-intensity in the diastolic phase is determined by not only microbubbles within capillaries but also by microbubbles contained in the arterioles and venules of the myocardium. Therefore, in our opinion, the more decreased myocardial video-intensity is during systole, the more decreased the capillary density in the region of interest on MCE. In the present study, ApHCM patients had significantly decreased myocardial video-intensity during systole, which means decreased blood volume and a high muscle-to-blood ratio of the hypertrophied segment. On the other hand, SPECT showed normal or rather increased perfusion of the apical segments. Nuclear tracer readily diffuses into and accumulates in the myocardium and increased uptake can occur in the hypertrophied segment of the LV of patients with ApHCM..$^{22,23}$ These results may represent abnormal capillary density and, probably, microvascular dysfunction occurring in the hypertrophied myocardial segments of patients with ApHCM, even without significant CAD. Although this characteristic change can be confirmed by histologic evaluation, the current diagnostic criteria for HCM (and ApHCM) do not justify practices such as an endomyocardial biopsy. Until now, there has not been an appropriate method of evaluating microvascular function in vivo. ${ }^{24} \mathrm{MCE}$ is a relatively economical, portable, noninvasive, and reproducible diagnostic modality for assessing microvascular dysfunction in HCM. Therefore, MCE could be a novel tool to evaluate abnormally reduced vascular density in the hypertrophied myocardium in HCM and provide helpful insights for better understanding the pathophysiologic changes of HCM.

\section{MCE Cyclic Variation, Perfusion Defect Size, and Contractile Function in ApHCM}

In this study, systolic mitral annular velocity (ie, S') in ApHCM patients was significantly decreased compared with the hypertensive LVH patients and normal subjects, whereas LVEFs were grossly comparable among the study groups. This means that the systolic longitudinal motion of the myocardium in ApHCM patients is more impaired than that of normal subjects and hypertensive LVH patients. Simultaneously, the degree of MCE cyclic variation had a significant positive correlation with $S^{\prime}$ in the entire study population and the perfusion defect size showed a significant negative correlation with S' in patients with ApHCM. Although it is not clear what these results mean, they are notable findings because decreased capillary density in the hypertrophied myocardial segment showed a significant correlation with the impaired systolic longitudinal motion reflected by TDI. In our opinion, the relatively decreased S' in ApHCM patients may represent an impaired myocardial systolic property and consequent subclinical systolic dysfunction in these patients, despite a normal LVEF. It was reported that TDI consistently detects myocardial dysfunction in patients with HCM without detectable $\mathrm{LVH}^{25}$ and even in HCM mutation carriers. ${ }^{26}$ Concurrently, experimental data strongly indicate the essential abnormality in HCM is impaired myocardial function, which provides the impetus for development of compensatory LVH. ${ }^{27}$ Therefore, myocardial systolic and diastolic functions would be expected to be impaired, even in the absence of segmental hypertrophy in HCM. The results of the present study tell us that this may be also relevant in ApHCM patients, because S' was determined at the septal and lateral corner of the mitral annulus, which were non-hypertrophied segments. Further study is warranted to reveal what a reduced S' means clinically in patients with ApHCM and its pathophysiologic association with the abnormal muscle-to-blood ratio in the hypertrophied segment of LV in these patients.

\section{Study Limitations}

First, our study had a small sample and second, coronary angiographic exclusion of CAD was not performed for every patient. Finally, histologic evaluation was not performed for ethical reasons. An animal study including histologic evaluation to determine the feasibility of MCE in HCM model is needed. 


\section{Conclusion}

MCE may detect an abnormal muscle-to-blood ratio in ApHCM patients. MCE may be a novel tool for evaluating reduced capillary density in the hypertrophied segment in patients with ApHCM and a helpful diagnostic test for better understanding ApHCM pathophysiology.

\section{Acknowledgment}

We thank the Medical Research Supporting Section of Yonsei University College of Medicine for providing excellent assistance with the statistical analysis.

\section{Disclosure}

None.

\section{References}

1. Kubo T, Kitaoka H, Okawa M, Hirota T, Hoshikawa E, Hayato K, et al. Clinical profiles of hypertrophic cardiomyopathy with apical phenotype: Comparison of pure-apical form and distal-dominant form. Circ J 2009; 73: 2330-2336.

2. Wigle ED, Rakowski H, Kimball BP, Williams WG. Hypertrophic cardiomyopathy: Clinical spectrum and treatment. Circulation 1995; 92: $1680-1692$.

3. Yamaguchi H, Ishimura T, Nishiyama S, Nagasaki F, Nakanishi S, Takatsu F, et al. Hypertrophic nonobstructive cardiomyopathy with giant negative $\mathrm{T}$ waves (apical hypertrophy): Ventriculographic and echocardiographic features in 30 patients. Am J Cardiol 1979; 44: $401-412$.

4. Cannon RO 3rd, Rosing DR, Maron BJ, Leon MB, Bonow RO, Watson RM, et al. Myocardial ischemia in patients with hypertrophic cardiomyopathy: Contribution of inadequate vasodilator reserve and elevated left ventricular filling pressures. Circulation 1985; 71: 234-243.

5. Maron BJ, Wolfson JK, Epstein SE, Roberts WC. Intramural ("small vessel") coronary artery disease in hypertrophic cardiomyopathy. $J$ Am Coll Cardiol 1986; 8: 545-557.

6. Maron BJ, Epstein SE, Roberts WC. Hypertrophic cardiomyopathy and transmural myocardial infarction without significant atherosclerosis of the extramural coronary arteries. Am J Cardiol 1979; 43: $1086-1102$.

7. Krams R, Kofflard MJ, Duncker DJ, Von Birgelen C, Carlier S, Kliffen M, et al. Decreased coronary flow reserve in hypertrophic cardiomyopathy is related to remodeling of the coronary microcirculation. Circulation 1998; 97: 230-233.

8. Jayaweera AR, Edwards N, Glasheen WP, Villanueva FS, Abbott $\mathrm{RD}$, Kaul S. In vivo myocardial kinetics of air-filled albumin microbubbles during myocardial contrast echocardiography: Comparison with radiolabeled red blood cells. Circ Res 1994; 74: $1157-1165$.

9. Lindner JR, Song J, Jayaweera AR, Sklenar J, Kaul S. Microvascular rheology of Definity microbubbles after intra-arterial and intravenous administration. J Am Soc Echocardiogr 2002; 15: 396-403.

10. Keller MW, Segal SS, Kaul S, Duling B. The behavior of sonicated albumin microbubbles within the microcirculation: A basis for their use during myocardial contrast echocardiography. Circ Res 1989; 65: $458-467$.

11. Chilian WM, Marcus ML. Phasic coronary blood flow velocity in intramural and epicardial coronary arteries. Circ Res 1982; 50: $775-781$

12. Goto M, Flynn AE, Doucette JW, Jansen CM, Stork MM, Coggins DL, et al. Cardiac contraction affects deep myocardial vessels predominantly. Am J Physiol 1991; 261: H1417-H1429.

13. Richardson P, McKenna W, Bristow M, Maisch B, Mautner B, O'Connell J, et al. Report of the 1995 World Health Organization/
International Society and Federation of Cardiology Task Force on the Definition and Classification of cardiomyopathies. Circulation 1996; 93: 841-842.

14. Lang RM, Bierig M, Devereux RB, Flachskampf FA, Foster E, Pellikka PA, et al. Recommendations for chamber quantification: A report from the American Society of Echocardiography's Guidelines and Standards Committee and the Chamber Quantification Writing Group, developed in conjunction with the European Association of Echocardiography, a branch of the European Society of Cardiology. J Am Soc Echocardiogr 2005; 18: 1440-1463.

15. Helak JW, Reichek N. Quantitation of human left ventricular mass and volume by two-dimensional echocardiography: In vitro anatomic validation. Circulation 1981; 63: 1398-1407.

16. Porter TR, Xie F, Kricsfeld D, Armbruster RW. Improved myocardial contrast with second harmonic transient ultrasound response imaging in humans using intravenous perfluorocarbon-exposed sonicated dextrose albumin. J Am Coll Cardiol 1996; 27: 14971501.

17. Matsubara K, Nakamura T, Kuribayashi T, Azuma A, Nakagawa M. Sustained cavity obliteration and apical aneurysm formation in apical hypertrophic cardiomyopathy. J Am Coll Cardiol 2003; 42: 288-295.

18. Eriksson MJ, Sonnenberg B, Woo A, Rakowski P, Parker TG, Wigle ED, et al. Long-term outcome in patients with apical hypertrophic cardiomyopathy. J Am Coll Cardiol 2002; 39: 638-645.

19. Maron BJ, Spirito P. Implications of left ventricular remodeling in hypertrophic cardiomyopathy. Am J Cardiol 1998; 81: 1339-1344.

20. Minoshima M, Noda A, Nishizawa T, Hara Y, Sugiura M, Iino S, et al. Endomyocardial radial strain imaging and left ventricular relaxation abnormalities in patients with hypertrophic cardiomyopathy or hypertensive left ventricular hypertrophy. Circ J 2009; 73: 2294-2299.

21. Toyota E, Fujimoto K, Ogasawara Y, Kajita T, Shigeto F, Matsumoto T, et al. Dynamic changes in three-dimensional architecture and vascular volume of transmural coronary microvasculature between diastolic- and systolic-arrested rat hearts. Circulation 2002; 105: 621-626.

22. Chu WW, Wallhaus TR, Bianco JA. SPECT imaging of apical hypertrophic cardiomyopathy. Clin Nucl Med 2002; 27: 785-787.

23. Cianciulli TF, Saccheri MC, Masoli OH, Redruello MF, Lax JA, Morita LA, et al. Myocardial perfusion SPECT in the diagnosis of apical hypertrophic cardiomyopathy. J Nucl Cardiol 2009; 16: $391-395$.

24. Kaul S. Assessment of coronary microcirculation with myocardial contrast echocardiography: Current and future clinical applications. Br Heart J 1995; 73: 490-495.

25. Nagueh SF, Bachinski LL, Meyer D, Hill R, Zoghbi WA, Tam JW, et al. Tissue Doppler imaging consistently detects myocardial abnormalities in patients with hypertrophic cardiomyopathy and provides a novel means for an early diagnosis before and independently of hypertrophy. Circulation 2001; 104: $128-130$.

26. Nagueh SF, McFalls J, Meyer D, Hill R, Zoghbi WA, Tam JW, et al. Tissue Doppler imaging predicts the development of hypertrophic cardiomyopathy in subjects with subclinical disease. Circulation 2003; 108: 395-398.

27. Marian AJ, Roberts R. The molecular genetic basis for hypertrophic cardiomyopathy. J Mol Cell Cardiol 2001; 33: 655-670.

\section{Supplementary files}

Movie 1. Myocardial contrast echocardiography in apical hypertrophic cardiomyopathy showing perfusion defect at the hypertrophied apical segment during systole.

Movie 2. Myocardial contrast echocardiography in apical hypertrophic cardiomyopathy (note the cyclic variation of video-intensity during cardiac cycles at the hypertrophied apical segment.

Please find supplementary file(s);

http://dx.doi.org/10.1253/circj.CJ-10-0241 